\title{
An Accelerated System for Melanoma Diagnosis Based on Subset Feature Selection
}

\author{
Ezzeddine Zagrouba ${ }^{1 *}$ and Walid Barhoumi ${ }^{2 \dagger}$ \\ ${ }^{1}$ Laboratory LIP2, Facultè des Sciences de Tunis, Tunisia \\ ${ }^{2}$ GRIFT, Laboratory CRISTAL, Ecole Nationale des Sciences de l'Informatique, Tunisia
}

In this paper we present an accelerated system for diagnosing skin lesions based on digitized dermatoscopic color images. This system is composed mainly of three levels : lesion detection, lesion description (features selection) and decision. The lesion detection level consists in the preprocessing of the lesion image in order to remove the undesired objects from the original image. Then, the extraction of the lesion is done by separating it from the healthy surrounding skin. The lesion description level is based on the extraction of a set of features modeling clinical signs of malignancy. The decision level is based on the produced vector of features scores, which is used as input to a multi-layer perceptron classifier in order to assign the lesion to the class of benign lesions or to the one of malignant melanomas. We focus particularly in this paper on the critical step of the features selection allowing to select a reasonable reduced number of useful features while removing redundant information and approximating the properties of melanoma recognition. This permits to reduce the dimension of the lesion's vector, and consequently the computing time, without a significant loss of information. In fact, a large set of features was investigated by the application of relevant features selection techniques. Then, the number of features for classification was optimised and only five well-selected features were used to cover the discriminatory information about lesions malignancy. With this approach, for reasonably balanced training/test sets, we record a good classification rate of $77.7 \%$ in a very promising CPU time.

Keywords: computer-aided diagnosis, melanoma, perceptron, feature subset selection, sequential floating search methods.

\section{Introduction}

The incidence of skin cancer is rapidly increasing through-out the world and it becomes one of the deadliest form of cancers specially in whitepopulation countries. Malignant melanoma is the third most frequent type of skin cancer and one of the most malignant tumors [3]. It is the least common but the most deadly skin cancer, accounting for only about $4 \%$ of all cases but $79 \%$ of skin cancer deaths [1]. Two main types of factors risk has been identified for the malignant melanoma: individual factors (numerous naevus, atypical naevus, reached antecedents) and a behavior factor which is the abused exhibition to the sun and to the artificial ultraviolets. Their incidence is on the order of $10-12$ per 100, 000 persons in Europe, 18-20 per 100, 000 persons in the United States of America and $30-40$ per 100, 000 persons in Australia [29]. Fortunately, if detected early, even malignant melanoma may be treated successfully. Many studies have clearly shown that the prognosis of malignant melanoma is directly depending on an early diagnosis followed by an appropriate surgical excision. However, the recognition of malignant melanoma is a difficult task, even for the trained dermatologists, since other skin lesions can have similar physical characteristics. A study at Karolinske hospital of Stockholm (Sweden), has shown that newly educated dermatologists with less than 1 year of experience

\footnotetext{
*Campus Universitaire, 1060 Tunis, Tunisia.

${ }^{\dagger}$ Campus Universitaire de La Manouba, Tunisia
} 
detect only $31 \%$ of the melanoma cases they are presented with, while dermatologists with more than 10 years of experience are able to detect just $63 \%[15]$ of the melanoma cases they are presented with.

Thus, seen the gravity of the melanoma presence and the doubt that reigns about its visual diagnosis, many dermatologists perform a medical procedure (called biopsy), which consists in appropriating a part of patient lesion, in order to ascertain whether the skin lesion is benign or malignant. The problem is that only $10 \%$ of these procedures reveal a cancerous pathology. It means that $90 \%$ people undergo a useless surgical intervention [34]. However, this operation which aims to avoid mistakes of diagnosis and it is no valid in most cases, involves some expenses and morbidity. It has some human effects, psychologic and physical (anesthesia), on the patients, particulary for those with multiple lesions, since it could cause unnecessary surgery. Besides, it has some material effects since it is necessary to pay for operations and hospitalization expenses.

For all these reasons, in recent years there has been a rising interest in the development of quantitative diagnosis support methods, with computer-aided diagnosis tools. In fact, the computer tool has one certain advantage on the human eye: a bigger perception of colors. Indeed, the human melanoma diagnosis is always done according to several criteria such as the color. However, human eye distinguishes two to three dozens of nuances of gray and about one thousand colors. The utilization of the digitized image in true colors permits the distinction of more than sixteen million colors and this number can progress very quickly with the fast evolution of the computer material. Thus, a computer uses other information that human eye cannot provide. Besides, with the automated data processing, it is possible to perform more deepened geometric and colormetric analyses of the lesion, subject of the diagnosis. It remains to note that the computer tool, that provides precious help to the clinicians, does not pretend to replace the physician. In fact, every doubt must be taken seriously into account, considering the gravity of the melanoma pathology.

Thus, over the last few years, much work has been done, producing useful computer-aided diagnosis systems for malignant melanoma recog- nition. The main objective of such systems is to assist dermatologists in different analysis steps, such as detection of the lesion boundary, quantification of diagnostic features, classification into different lesions types, visualization, etc [29]. In the following section, we will present an overview of the literature of computer-aided melanoma diagnosis systems based on image processing techniques. We also present an accelerated system for melanoma diagnosis. In section 3, we present the architecture of our system (Figure 1) while retailing the lesion detection, the lesion description and the decision levels. The lesion detection level consists of preprocessing the lesion image followed by the extraction of the lesion by separating it from the healthy surrounding skin. The lesion description level is based on the feature extraction process and on a subset feature selection technique. This permits to define a pertinent descriptor vector relatively to the lesion. This lesion's vector will be then the raw input to a multi-layer perceptron classifier (decision level) in order to assign the lesion to the malignant melanoma class or to the benign nevi class. In section 4, we will discuss the relevance of our proposed system by analysing results of the experiments in terms of true-positive and true-negative rates, hence permitting to evaluate the global classification accuracy of our diagnosis system. Finally, conclusion and suggestions for future work are given in section 5 .

\section{Overview of the Literature}

This section presents a review of the existing literature on computer-aided diagnosis systems for melanoma recognition. A classic system of melanoma diagnosis is principally composed of three stages. First, after the acquisition and the preprocessing of the dermatoscopic image, the segmentation process aims to extract the lesion $L$ from the Healthy Surrounding Skin $(H S S)$. Then, the lesion is described by a set of photometric and geometric attributes, modeling clinical signs on the lesion malignancy. Finally, classification is performed in order to determine the type of the lesion, relatively to the determined attributes set. In the following we present existing computer-aided melanoma diagnosis systems. 
In a previous work [34], we presented a prelimary approach for the automated recognition of malignant melanoma. In order to localize suspicious lesion regions, we introduced an automated approach to segmentation by region growing, after a preliminary step based on fuzzy sets. Then, relatively to every detected skin lesion, we measured a series of attributes modeling the clinical malignancy symptoms. Finally, the selected attributes were supplied to an artificial neural network in order to differentiate melanomas from benign lesions. This approach allowed us to obtain $79.1 \%$ of correct classification of malignant and benign lesions on a test set consisting of 200 real skin lesions images.

In [15], Hintz-Madsen developed an efficient system for diagnosing skin lesions images. $\mathrm{He}$ used an optimal thresholding technique to determine two thresholds separating the light skin background from the dark skin lesion. In the case that the segmentation produces several skin lesion candidates, due to other small non-lesion objects, the largest object is selected as the skin lesion. To realize the lesion classification, a neural network in a probabilistic framework was designed and it allowed to detect $73.2 \%$ of benign skin lesions and $75 \%$ of malignant skin lesions on a test set of 200 images.

In [30], the authors have introduced a knowledgebased system for supporting the early diagnosis of melanoma. It detects the lesion using a thresholding technique applied on the blue and on the saturation images. Then, the system, called MEDS (MElanoma Diagnosis System), extracts a set of colorimetric and geometric features. It then yields a diagnosis based on a voting schema integrating the results produced by three different classifiers: k-nearest neighbors $(\mathrm{KNN})$, discriminant analysis and decision tree. The system is trained and validated on a set of 152 skin images. The average sensitivity and specificity of the designed system were $82 \%$ and $71 \%$ respectively.

In [29], the authors presented a preliminary computer-aided diagnosis system for pigmented skin lesions, with solutions for lesion boundary detection and for the quantification of the asymmetry degree. To detect the lesion boundary, they used a segmentation technique based on the clustering of a two dimensional color space. To perform the clustering, a modified Fuzzy CMeans (FCM) technique (called Orientation-
Sensitive FCM: OS-FCM) was introduced. Lesions detection results were validated by expert dermatologists, who also provided hand-drawn boundaries of the lesions. The authors showed that dermatologists were not able to reproduce their results. In particular, the boundaries of any expert taken alone showed higher divergence from those of remaining experts as well as from the developed automatic techniques. In this paper feature extraction is restricted to the quantification of degree of symmetry by a sixdimensional vector, which was used to classify, with a linear classifier, pigmented skin lesions as being benign or malignant. This classifier achieved a sensitivity of $60 \%$ and a specificity of $70 \%$ on a test set of 100 skin lesion images.

Vannoorenberghe et al [33] proposed a color image segmentation method based on the DempsterShafer theory. The tristimuli Red, Green and Blue are considered as three independent information sources, imprecise and uncertain, permitting separation of the pigmented lesion from the healthy surrounding skin by computing a threshold obtained by means of the Maximum Entropy Principle (MEP). Then, the three relative decisions are combined by the DempsterShafer theory technique. In the second step, features concerning the lesion are extracted using color information and all these primitives are considered as uncertain information sources on the lesion malignancy. Then, these informations are combined using Dempster-Shafer theory to bring out a particular behavior for the malignant melanoma. This classification procedure was applied on a set of 120 skin lesion images with a general success rate of $63 \%$.

In [22], the authors proposed a similar classification method based on the Dempster-Shafer theory and information criteria. In fact, after an original basic belief assignment with probability densities obtained by learning, an attenuation factor based on the dissimilarity between probability densities is introduced. The proposed method allows to obtain $81.1 \%$ of good classifications. However, the major drawback of this method resultes in increased CPU time.

Ercal et al [7] presented a neural network approach for the automated separation of melanoma from three benign categories of tumors, which exhibit melanoma-like characteristics. The approach uses discriminant features, based on tumor shape and relative tumor color, that are 
supplied to an artificial neural network for classification. This approach permitted to obtain above $80 \%$ correct classification of malignant and benign tumors on real skin tumor images.

Ganster et al [12] developed a system to enhance an early recognition of malignant melanoma. As an initial step, the binary mask of the skin lesion is determined by several basic segmentation algorithms, which are combined together with a fusion strategy. A set of 122 features containing shape and radiometric features, as well as local and global parameters, was calculated to describe the malignancy of a lesion. Using statistical feature subset selection methods, 21 significant features were selected from this set. Finally, a k-nearest neighbors classifier is used for the classification with a sensitivity of $81 \%$.

Some other non-complete systems were also introduced. For example, in [8], the authors used color-based segmentation approach applied on the Karhunen-Loève transform of the rgb color vectors to separate pigmented lesions from the skin and proceed with histogram equalisation and greyscale morphology to enhance and filter the pigmented lesions. They demonstrated that this approach allows to enhance pigmented skin lesions visually, and make them accessible to further analysis and classification tasks.

In [28], the authors introduced a region-based approach to segment lesion images into areas of different colors. Initially, the process divided the dermatoscopic image into rectangular regions, small enough to be considered as having only a single color. This is followed by conservative merging, where adjacent regions whose colors are very similar are coalesced. The segmentation is then completed by an iterative optimal merging process, the two most similar regions being merged at each step until the stopping condition is reached.

In 1995, Lee et al. [20] presented an algorithm to identify skin lesions from the digitized color images. After the application of a multi-stage median filter on the original image, in order to suppress the noise, the algorithm consists of the computation of threshold values for the lesions and the background normal skin. Then, the application of a rule-based system permits identification of the lesions.

In [14], the authors defined an approach by adaptive thresholding for the automated segmenta- tion of color skin lesion images. This approach combines six different thresholding techniques which are applied on the principal component of the Karhunen-Loève transform, on the rgb space and on the spherical coordinates.

Finally, we can conclude that many existing systems produced high rates of malignant melanoma recognition. But, their major inconvenience is too high computing time. The use of more than one algorithm for segmentation and/or classification to be combined, and the use of a large set of features, is the main cause of the non-realistic computing time. In many works [34] [29], it was confirmed that the task of detection of a specific pattern in dermatoscopic images is both, the most interesting and very difficult. In other words, computing the features that model the clinical signs of malignancy is the step with the largest CPU time consumption in a computeraided melanoma diagnosis system. In fact, a fundamental problem of automated melanoma recognition is the complexity of the classification scheme, because a large number of features can be found in the feature design process. In order to keep the complexity low, a reasonable number of useful features, that remove redundant information and approximate properties of the melanoma diagnosis task, should be selected. A general theory for the design of such features does not exist [11]. However, reduction of the feature set dimension is very important and must be addressed in the right way. On the one hand, smaller feature set reduces the cost of the measurements. On the other hand, reduction in the number of features may lead to a loss of the discriminatory power of the classification and consequently may reduce the accuracy of the resulting system.

Roß et al. [27] performed selection of features by application of the "sequential forward selection algorithm" that reduced the set of features, starting with 87 features, to five features. In [15], the authors achieved feature selection with a neural network by application of node pruning. This approach allowed to reduce the set size from 21 lesion features to only six. However, Ganster et al. [12] started with 122 features and used three statistic approaches to reduce the number of the selected features. These approaches are: Sequential Floating Forward Selection (SFFS), Sequential Floating Backward Selection (SFBS) and Leave-One-Out (LOO). 
Finally, a subset size of 21 is chosen for further classification experiments.

\section{The Proposed System}

In this section we will establish the scheme of our proposed computer-aided diagnosis system for skin lesions recognition. This system is presented in Figure. 1 and is composed mainly of a lesion detection, a lesion description and a decision levels. The input to our system are color images ( $\mathrm{rgb}$ ) which are digitized photographic slides of dermatoscopic images [34]. The lesion detection level consists of the realization of two steps. The first step concerns the preprocessing of the lesion image in order to eliminate the noise and the undesired objects in the original image. The second step consists of the extraction of the lesion by separating it from the healthy surrounding skin. This step produces a binary image (lesion $v s$. healthy surrounding skin) which will be treated by the border extraction process, in order to define a polygon representing an approximation of the lesion boundary. The lesion description level is based on the realisation of two steps. The first step is the features extraction process producing numerical features which designate clinical symptoms of malignancy. Then, in order to remove redundant information and approximate the properties of melanoma recognition, selection of features allows to select a reasonably reduced number of useful features, that permits to reduce the dimension of the lesion's vector, and consequently the computing time, without significant loss of information. Finally, the decision level consists of the presentation of this lesion's vector as an input to a multi-layer perceptron classifier in order to assign the lesion either to the class of malignant melanoma or to the class of benign lesions.

Finally, let's note that the proposed system represents an extension of the system presented in [34]. Indeed, here our interest will be focused on the step of features selection which represents the main amelioration in our system. This step will allow to reduce considerably the computing time of our computer-aided diagnosis system without significant loss of the classification performance.

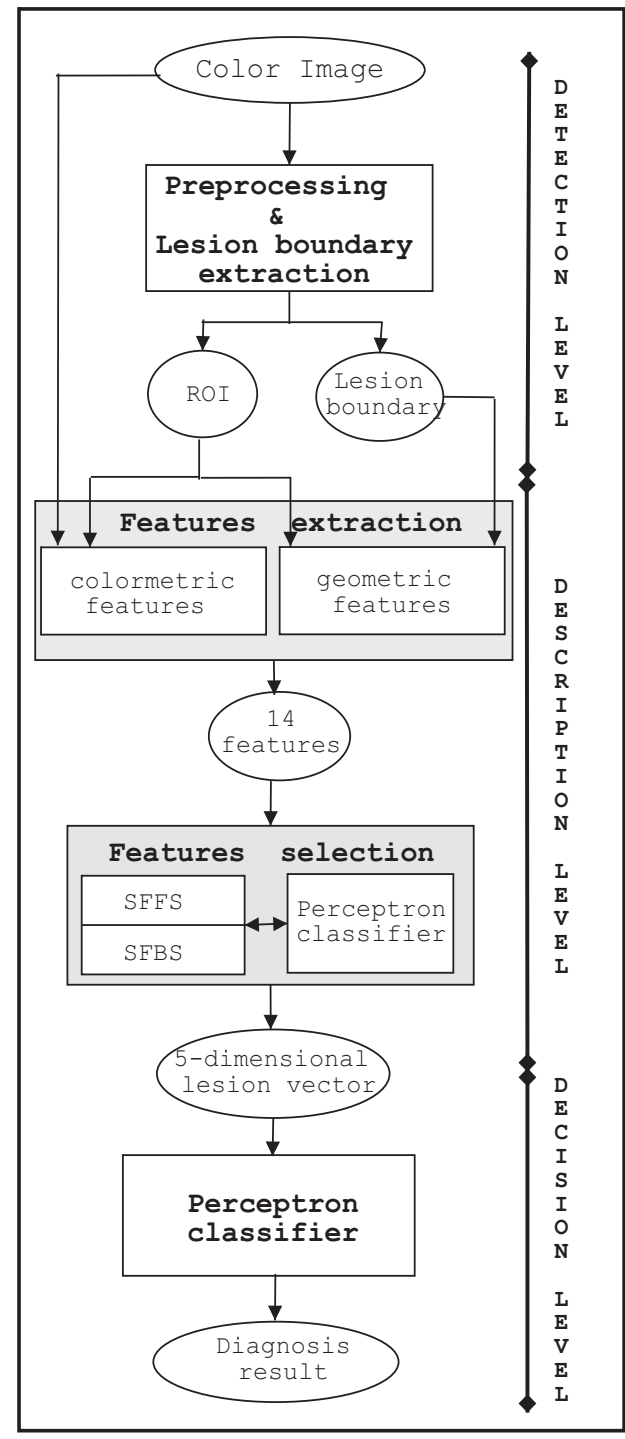

Fig. 1. Architecture of the proposed system.

\subsection{Lesion Detection Level}

The first step of the lesion detection level is the preprocessing of the dermatoscopic original image aiming to ameliorate the image quality for ulterior tasks. The second step is based on the preprocessed image and it aims to define the lesion by a region of interest (ROI) and to extract the correspondent boundary.

\subsubsection{The Original Image Preprocessing}

The original image preprocessing consists of the removal of everything that might corrupt the image and hence the ulterior segmentation steps results. One of the most undesired objects that are always present in dermatoscopic 
color images are impulsive-like artifacts. These artifacts can be considered as impulsive noise and may thus be reduced using a median filter [13] while preserving edges. The median filter is very effective in removing noise spikes that only cover a few pixels compared to the kernel neighborhood size. Thus, it can remove blond hairs and small air bubbles from skin images (Figure 2.c). Besides, as we were working with color images, we applied the median filter to all three color components ( $\mathrm{r}, \mathrm{g}, \mathrm{b})$ separately.

The next preprocessing step aims to facilitate the segmentation process by enhancing the edges in the image. For this purpose, we applied the Karhunen-Loève (KL) transform [23] on the resulting filtered images in order to enhance the edges which permits eventually to ease the separation between the lesion and the surrounding healthy skin. The Karhunen-Loève transform is a linear transform that correlates the input variables by employing an orthonormal basis. This basis is found by an eigenvalue decomposition of the sample covariance matrix of the input variables. The covariance matrix $\mathrm{Cov}$ is computed with a vector $V$ enclosing all three color vectors of the image. In fact, we use projections of the color vectors onto the eigenvectors of the covariance matrix that models the variation of the color components. Due to the decreasing ordering of the eigenvalues and the corresponding eigenvectors, the first principal component will contain the maximum variance [34]. Thus, since most variations occur at the edges between the lesion and surrounding skin, the first principal component is a natural choice for further segmentation (Figure 2.d).

Besides, some skin images contain dark hairs with similar color hue to the lesion, which may occlude the lesion and mislead the segmentation

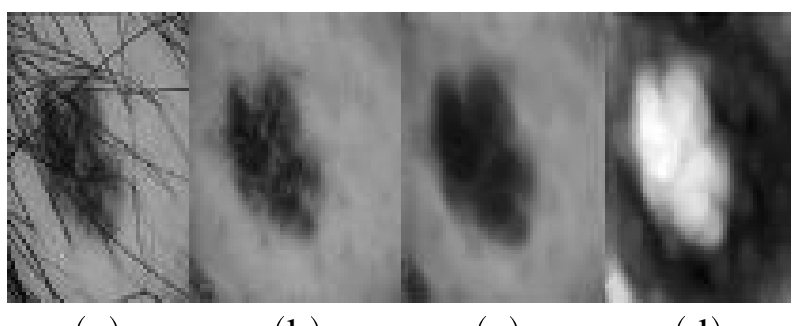

(a)

(b)

(c)

(d)

Fig. 2. Image preprocessing. (a) original image; (b) hairs removal; (c) median filtering; (d) first principal component of the Karhunen-Loève transform. process. In fact, while blond hairs can be left without problem with the median filter, this filter is insufficient for dark hairs suppression and can even intensify some undesired dark hairs (Figure 3.). To remove these dark hairs from the dermatoscopic image, we implemented the program solution called "DULLRAZOR" [21] proposed by Lee et al. This solution consists of identifying the dark hair locations by applying a generalized morphological closing operation to the three color bands separately, and replacing the determined hair pixels with the nearby no-hair pixels [34] (Figure 2.b).

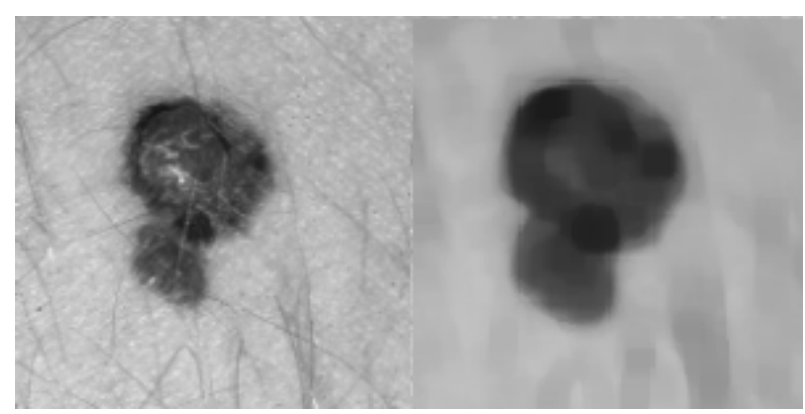

(a)

(b)

Fig. 3. Insufficiency of median filter for dark hair removal. (a) original image; (b) median filter result.

\subsubsection{The Lesion Boundary Extraction}

The lesion boundary extraction is a difficult task in dermatoscopic images since the transition between the lesions and the surrounding healthy skin is often smooth and ill-defined. Indeed, most skin lesions possess fuzzy boundaries with slow and extended transition from the dense core region to the surrounding tissues. In the literature, various region-based segmentation methods are applied for the skin lesion detection and the most commonly used technique has been color and grayscale thresholding [18]. However, the majority of these methods are unable to define the criterion to precisely separate pigmented lesion from the surrounding healthy skin.

To accomplish detection of the skin lesion, we used our automated approach of segmentation, introduced in [34]. It consisted of performing a region growing algorithm after the preliminary step, which is based on fuzzy sets. This preliminary step allows generation of a fuzzy image which is based on gray level mapping into a 
fuzzy plane, using a membership transformation function. The goal is to generate an image of higher contrast than the input image (the first principal component of the median filtered dermatoscopic image) by giving a larger weight to the gray levels that are closer to the lesion representative gray level than to those that are farther from the lesion representative gray level.

In fact, after a succession of intensity histogram equalizations, we obtained a bimodal curve characterized by two peaks $p_{1}$ and $p_{2}$ and their corresponding gray levels $g_{1}$ and $g_{2}$ assumed to be representative of the gray levels of the lesion and the surrounding healthy skin. Then, as the lesion is always darker than the surrounding skin, its gray level $g_{L}$ shall be the minimum of $g_{1}$ and $g_{2}$. Given the lesion gray level $g_{L}$, a pixel whose gray level is similar to $g_{L}$ could be assigned a high intensity in the resulting fuzzy image. To perform the enhancement of this fuzzy image an appropriate membership transformation function, that evaluates the similarity between the gray level $g l(p i x)$ of the pixel pix being considered and the representative gray level $\left(g_{L}\right)$ of the lesion, may be achieved. This function must be symmetric, it decreases monotonically from 1 to 0 and assigns a membership degree equal to 1 to those pixels that possess the same gray level as the lesion $\left(g_{L}\right)$. We have considered two functions, $\Im 1$ (eq. 1) and $\Im 2$ (eq. 2), verifying these properties.

$$
\begin{gathered}
\Im 1(\text { pix })=\left(1+\beta * \mid g l(\text { pix })-g_{L} \mid\right)^{-1} . \\
\Im 2(\text { pix })=1-\beta * \mid g l(\text { pix })-g_{L} \mid .
\end{gathered}
$$

We deduce that better results are obtained with a normalized combination, $\Im$ (eq. 3 ), of these two functions $\Im 1$ and $\Im 2$ (Figure 4.). Finally, the resulting fuzzy matrix could be used as a scale factor to obtain gray levels and display the resulting fuzzy image $I_{f u z z y}$ (eq. 4).

$$
\begin{gathered}
\Im(\text { pix })=\frac{\Im 1(\text { pix })+\Im 2(\text { pix })}{2} . \\
I_{f u z z y}(\text { pix })=\Im(\text { pix }) \cdot \max _{p \in \Im} g l(p)
\end{gathered}
$$

Value $\beta$ defines opening of the membership function. For large $\beta$ the opening is narrow and the function behavior is strict. However, for small $\beta$ the opening is wide, and the function

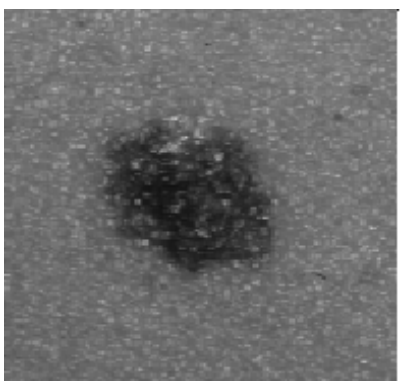

(a)

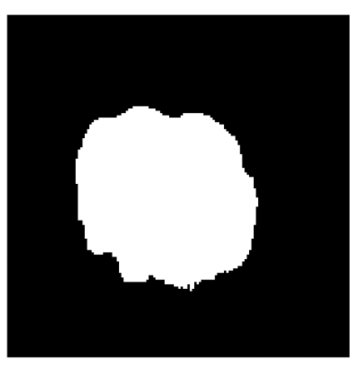

(c)

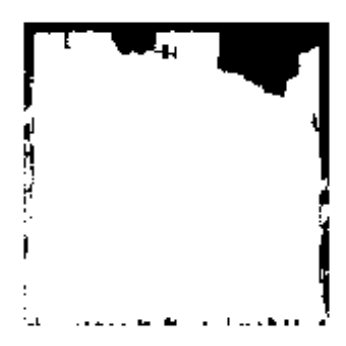

(b)

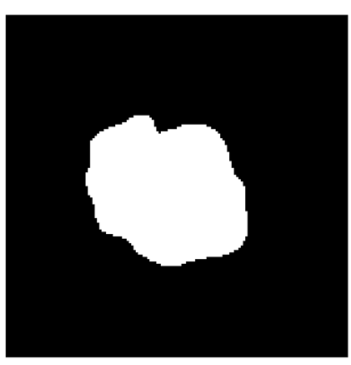

(d)
Fig. 4. Comparison between the membership functions results. (a) original image; (b) lesion detection with the function $\Im 1$; (c) lesion detection with the function $\Im 2$; (d) lesion detection with the function $\Im$.

presents a more permissible behavior (Figure 5., Figure 6.).

To perform the region growing algorithm, a representative seed pixel must be defined inside the skin lesion. This seed pixel $g$ is defined by the pixel having $g_{L}$ as intensity while being the center of a homogeneous neighborhood $V(g)$ (eq.5). Finally, starting from the determined seed pixel, a classic region growing algorithm [13] is applied on the resulting fuzzy matrix and it permits to define precisely the lesion as a re-

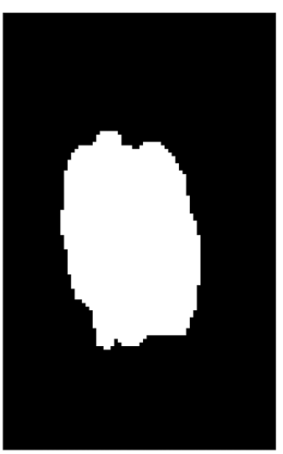

(a)

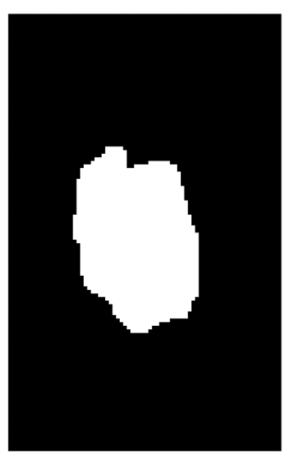

(b)
Fig. 5. Depending on lesion extraction relatively to $\beta$. (a) $\beta=0.006$; (b) $\beta=0.011$ (binary plane relative to the lesion in Figure. 4.(a). 


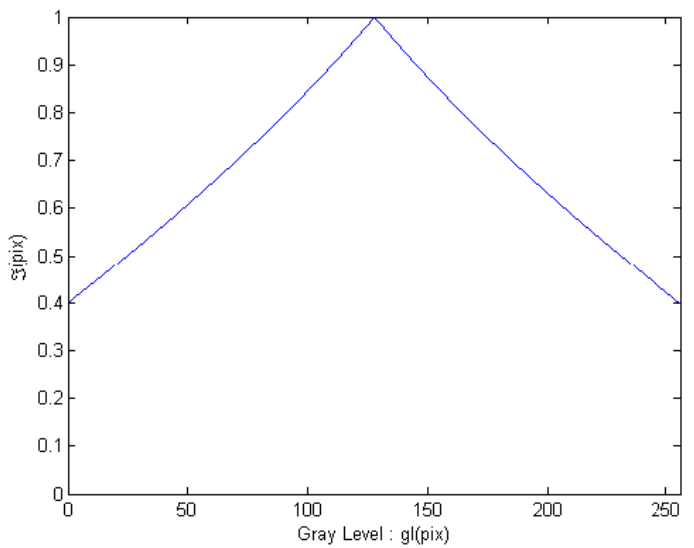

(a)

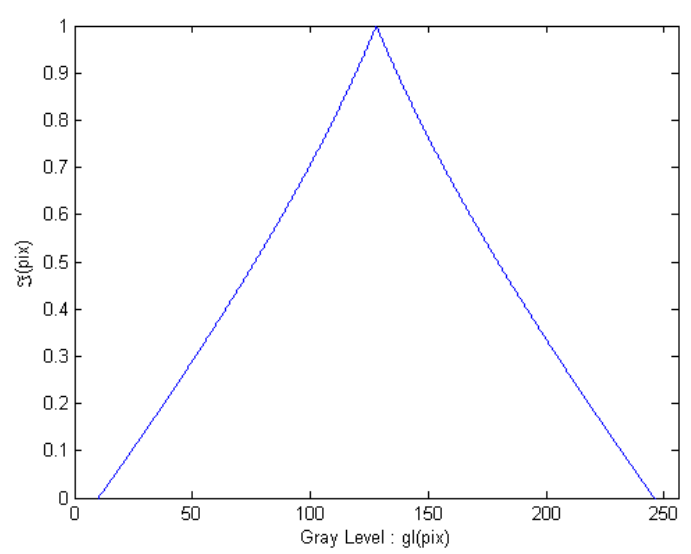

(b)

Fig. 6. Depending on the function $\Im$ behavior relatively to $\beta\left(g_{L}=128\right)$. (a) $\beta=0.006$; (b) $\beta=0.012$.

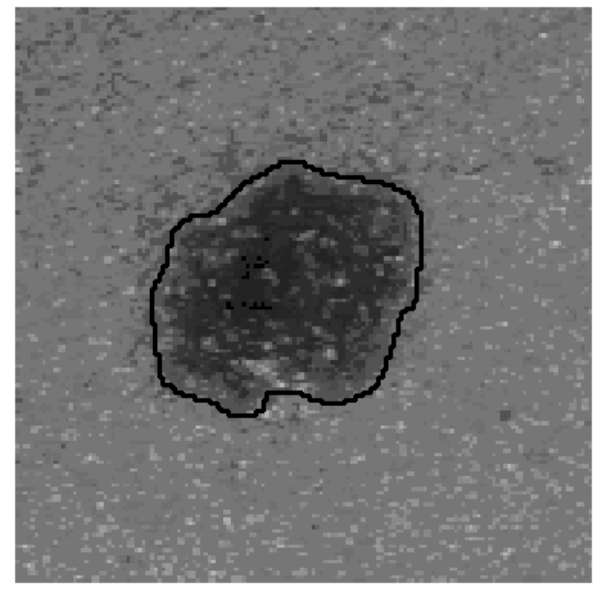

Fig. 7. Detection of the lesion boundary of the lesion in Figure 4.(a).

gion of interest (ROI). The output of the region growing procedure is the so-called binary plane (Figure 5.), i.e. 1-bit image that separates lesion
(1) from the healthy surrounding skin $(0)$. The resulting binary image (lesion vs. healthy surrounding skin) is then treated by an algorithm of follow-up applied on the border pixels of the lesion which are characterized by a local maximum of gradient. This allows the definition of a closed boundary relating to the lesion (Figure 7.).

$$
\begin{aligned}
\max _{q \in V(g)} & |g l(g)-g l(q)|= \\
& =\min _{p \in \xi}\left[\max _{q \in V(p)}|g l(p)-g l(q)|\right] .
\end{aligned}
$$

where, $\xi=\left\{p \in \Im / g l(p)=g_{L}\right\}$.

\subsection{Lesion Description Level}

The first step of the lesion description level is based on the binary plane defining the skin lesion by a region of interest and on the corresponding boundary of the lesion which is produced by the lesion detection level. This step consists of the extraction of a set of numerical features modeling clinical signs on the lesion malignancy. Then, in order to remove redundant information and to approximate the properties of melanoma recognition, a features subset selection step allows to select a reduced number of features. This step allows to reduce the dimension of the lesion's vector without losing information about the lesion malignancy.

\subsubsection{Lesion Features Extraction}

The objective of the lesion features extraction step is to quantify clinical signs used by a dermatologist for the melanoma diagnosis by a set of $m$ numerical features. Thus, given its definition by a region of interest (ROI) and by a closed boundary, every lesion will be described by a $m$ dimensional feature vector. The extraction of these features is based on the so-called $A B C D$ rule of dermatoscopy. This rule, originally introduced by Friedman et al. [9], is a very useful tool in facilitating the malignant melanoma diagnosis. It analyses four clinical characteristics to identify a malignant melanoma. These characteristics are the lesion Asymmetry, the lesion Border irregularity, the Color variation inside the lesion and, finally, the lesion Diameter. 
In the literature, many attributes were used to describe characteristics of the lesions malignancy relatively to the $A B C D$ rule. However, as it was always proved [29], a unique attribute is not sufficient to diagnose precisely melanoma cancer. Combination of different criteria is the key to an early detection of malig-
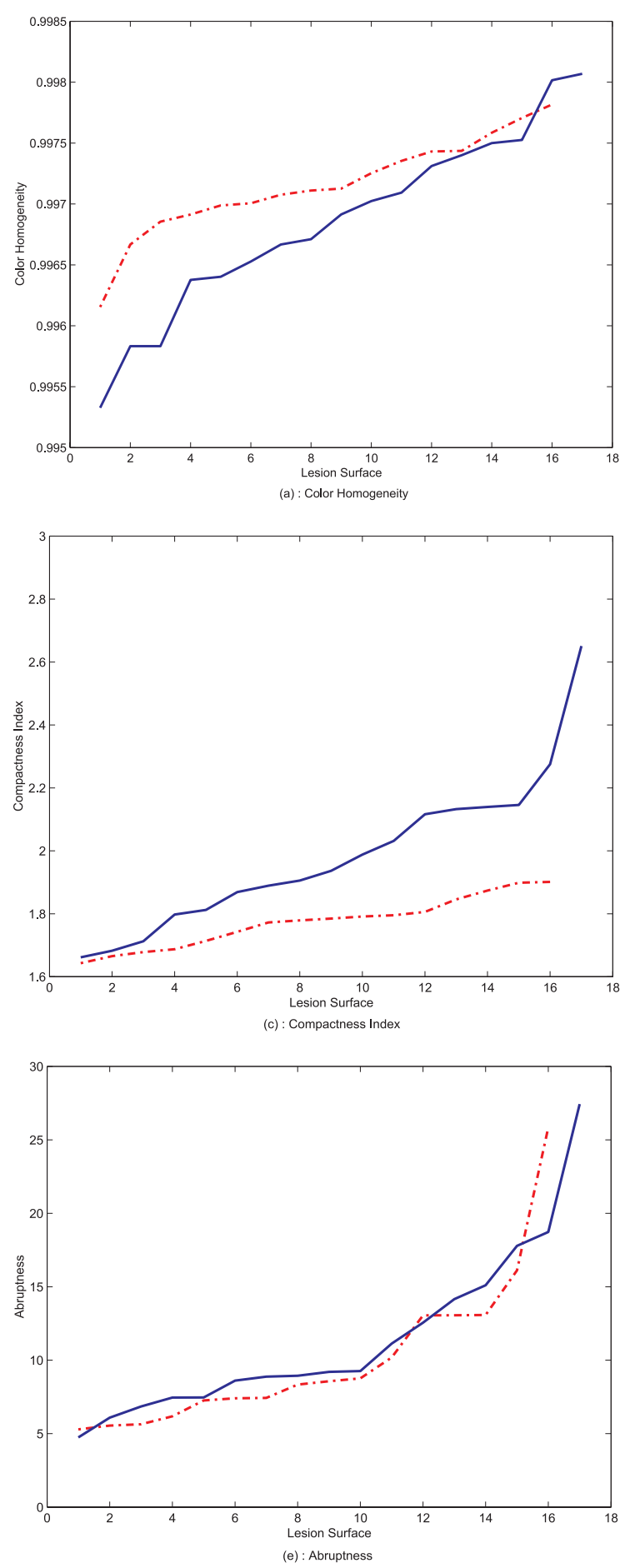

nant melanoma. In this sense, we carried out a study of many significant attributes, according to the surface of the lesion. The means of these parameters per interval of lesions surfaces were calculated on randomly selected images of malignant and benign lesions (Figure 8). We deduced an important correlation between most
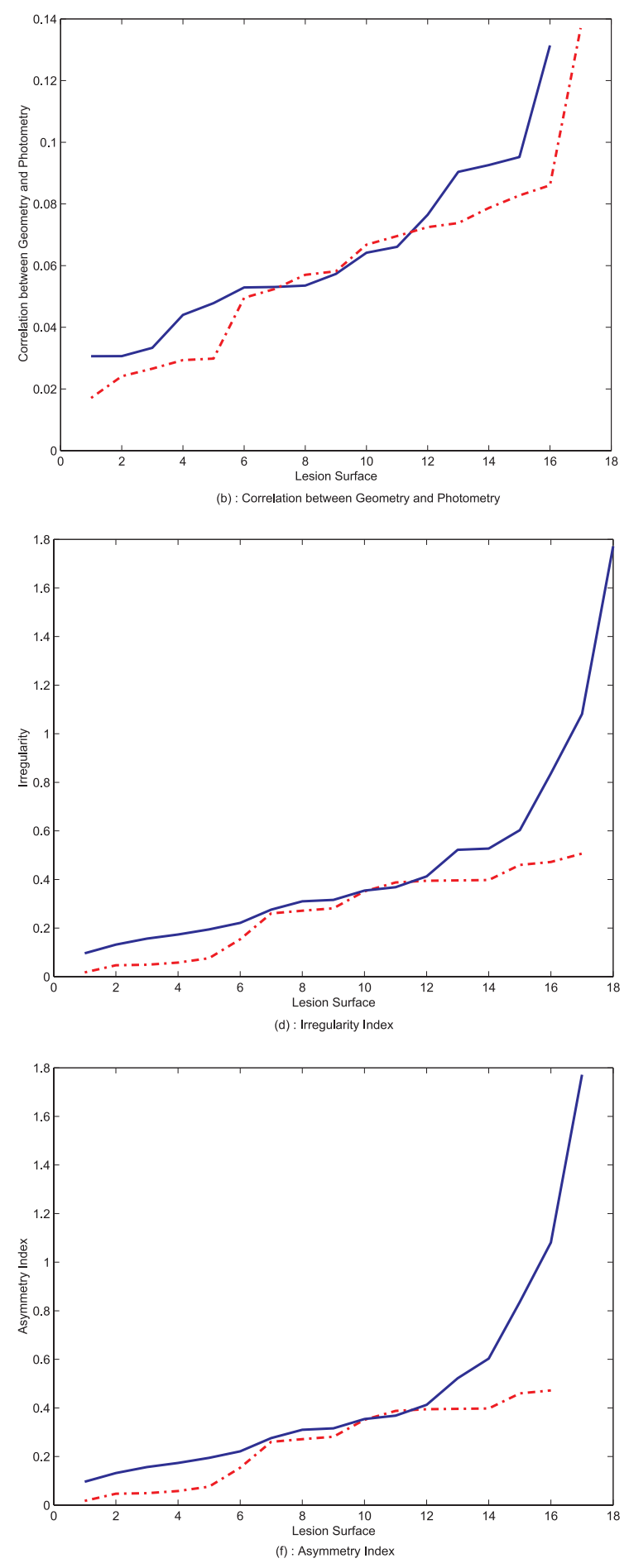

Fig. 8. Distribution of some attributes relatively to malignant ' - ' and benign ' - - ' lesions. 
of the attributes and the melanoma diagnosis. The two types of lesions differ slightly in their characteristics relatively to these attributes. For example, consider the color homogeneity attribute (Figure 8.a.). The value of this attribute is normally more elevated for the benign lesions that for the malignant ones. However, it is often possible to encounter malignant lesions of which the value of the color homogeneity attribute is more elevated than the ones recorded with some benign lesions and the same behavior can be deduced with other attributes (Figure 8.). Thus, we can conclude that it is impossible to separate linearly the two types of lesions using every attribute separately. From there, it is necessary to perform a combination of a set of suitable attributes in order to obtain precise classification of the lesion.

As the next step, we will perform feature selection by choosing to use a relatively large set of possible attributes. After literature overview of the more used attributes to quantify the $A B C D$ rule and after many discussions with trained dermatologists, we selected 14 attributes modeling the lesion malignancy symptoms used by experts in clinical practice. These attributes are [34] [7] the asymmetry index, the lesion diameter, the lengthening index, the compactness index, the mean square error between the image and its reflected version, the fractal dimension, the edge abruptness, the mean and the variance of the gradient magnitude along the lesion boundary, the color homogeneity, the relative chromaticity of the red, green and blue components and finally the index of correlation between geometry and photometry.

\subsubsection{Features Subset Selection}

The goal of this step is, given the lesions set described by $m(m=14)$ attributes, to find the minimum number $n(n<<14)$ of relevant attributes which describe the lesions malignancy as well as the original set of attributes do [19]. In fact, there is often a need to minimize the number of attributes actually used for classification, since each used attribute increases the running time of the computer-aided diagnosis system. At the same time, there is a need to achieve high recognition rates. This has led to the development of a solution for finding an "optimal" subset of features from a larger set of possible features. The produced subset of features would achieve high melanoma recognition rates, while at the same time it decreases cost and running time of the computer-aided diagnosis system.

In the literature, two main categories of features selection are used [32]. The first approach selects features independently of their effect on the classification performance. However, mere evaluation of the discriminatory power of the initial $m$ features individually is not sufficient, but all subsets must be considered to find the best combination [5]. Thus, the second approach, called subset features selection, selects directly a subset of the available $m$ features, without significant decrease of the recognition performance. Several algorithms are used for the realization of the second approach and as the complexity of the algorithm grows exponentially with $m$ [26], it is not possible to derive the optimal performance by exhaustive search and all these algorithms are sub-optimal. Besides, we note that the feature selection process is always followed by a measure of the discriminatory power of subsets given a separability criterion, which measures the discriminatory power of the subset under consideration.

Given the dimension of the desired subset of features, probably the most effective feature selection techniques are the sequential floating search methods (SFSM) [16] [25]. There are two main categories of floating search methods: forward and backward. In the case of sequential floating forward search (SFFS), the algorithm starts with a null feature set and, for each step, the best feature satisfying some criterion function is included with the current feature set. Besides, at every step, the worst feature concerning the criterion is eliminated from the set. Therefore, the SFFS proceeds dynamically, increasing and decreasing the number of features, until the desired set dimension is reached. The backward search (sequential floating backward search: SFBS) works analogously, but starting with the full feature set and performing the search until the desired dimension is reached. These algorithms have been improved, leading to the adaptive floating search methods (AFSM) [31]. The main difference is in the number of desired features that can be determined dynamically. In [4], the authors proved that the performance of AFSM was very similar to the performance of SFSM. However, the AFSM needed much more computing time. 
In this work, we perform an automatic subset features selection technique where subsets of the initial set of 14 attributes are investigated in order to derive a combination of a reduced set of pertinent attributes modeling the $A B C D$ rule. To do this, we applied two sequential search techniques: Sequential Floating Forward Selection (SFFS) and Sequential Floating Backward Selection (SFBS). These algorithms use stepwise inclusions and exclusions of features into/from the subset of consideration. As separability criterion, we used the performance of a multi-layer perceptron classifier using the selected features. We performed the SFFS and SFBS techniques with various size of the designed features subset. Our experiments showed that the best performance is recorded with a subset of five features determined by the SFFS technique and the correspondence between the selected features and the clinical criteria is clearly shown (Figure 9).

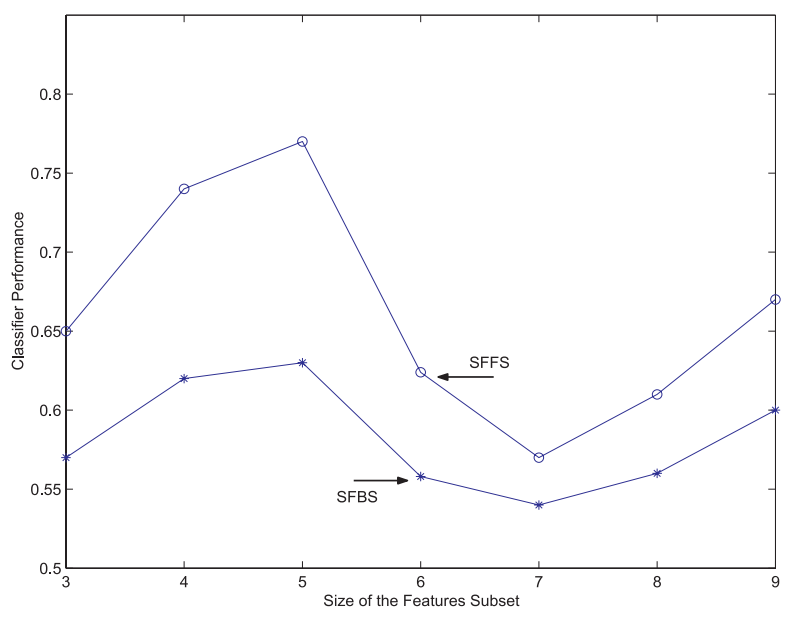

Fig. 9. Results of subset feature selection with SFFS and SFBS algorithms.

\subsection{Decision Level}

The final level of our melanoma computeraided diagnosis system is the lesion screening decision, looking for the association between the studied skin lesion and the malignant melanomas class, or the benign lesions class. This level is based on the supplying of the lesion's vector, produced by the previous level, to a multi-layer perceptron classifier. In the literature, many classical methods such as the nearest neighbors classification [12], fuzzy c-means $(\mathrm{FCM})[6]$ as well as neural networks [11] [30]
[7], have been applied for the purpose of skin lesions classification. However, it was always showed that the recognition rates are slightly higher for the neural networks classification, compared to the classical methods [11]. Thus, to perform the classification procedure, as neural network classifier we used a multi-layer perceptron trained with the standard back-propagation algorithm [10]. The input to this perceptron is the lesion's vector produced by the previous level. However, as the features composing the lesion's vector have different unit and different value ranges, an objective scaling, between 0 and 1 , of the feature values is achieved by calculating the correspondent $z$-scores [17]. Therefore, given a feature $j$ and the set of $t$ samples ( $t$ is the size of the training or the test sets) for the feature $j$, the $i^{t h}$ sample measure of feature $j$ $\left(X_{i j}\right)$ will be replaced by the value $Y_{i j}$ given by:

$$
Y_{i j}=\frac{X_{i j}-\overline{m_{j}}}{\sigma_{j}} .
$$

where, $\overline{m_{j}}$ is the mean value of the $t$ samples for the feature $j$ and $\sigma_{j}$ is the correspondant mean absolute deviation.

The perceptron architecture is formed of only one hidden layer [34], whose number of units is determined manually, by evaluating the classification performance (Table. 1.). The output layer consists of one linear output unit producing a binary result ( 1 for a malignant lesion and 0 for a benign lesion) which corresponds to the target class. The defined perceptron is trained with supervision, given a training set of dermatoscopic images, using the standard backpropagation algorithm [10].

\section{Tests and Results}

Table. 1. illustrates the results obtained using the designed multi-layer perceptron, with 14 input units representing the 14 features introduced in the subsection 3.2.1., for different numbers of hidden units so that the effect of the perceptron architecture on the classification performance could be assessed. It turned out that the best recognition rates, both on the training set and on the test set, could be achieved by using four hidden units. The training process time was about 50 minutes for error below 0.1 and a set of 10.000 iterations was executed. 
This architecture allows to obtain above $95 \%$ of good classifications on the training set and above $82 \%$ of good classifications on the test set.

\begin{tabular}{lll}
\hline hidden units & training set $(\%)$ & test set $(\%)$ \\
\hline 1 & 78 & 73.7 \\
\hline 2 & 75.7 & 72.5 \\
\hline 3 & 84.9 & 74.6 \\
\hline 4 & 95.3 & 82.4 \\
\hline 5 & 91.8 & 78.1 \\
\hline
\end{tabular}

Table 1. Classifier performance with reference to employed hidden units.

The main inconvenience of this classifier is its higher calculating time caused mainly by the large number of features used to describe a lesion. Thus, the legitimate solution for reducing the complexity of the classification process was to reduce the number of features without losing information about the malignancy of the lesion. Hence, we used the feature subset determined by the SFFS technique permitting decrease of the size of feature set from 14 to 5 while preserving similar significant information on the lesion malignancy. In fact, a recognition rate of $77.7 \%$ is achieved with five features and it does not increase over $82.4 \%$ by including the 14 features. However, decreasing the size of the feature set allows to reduce considerably the computing time for both, features computing $\left(t_{F C}\right)$ step and classification step, including the training $\left(t_{T 1}\right)$ and the test $\left(t_{T 2}\right)$ modes (Table. 2.).

\begin{tabular}{llll}
\hline features set dimension & $t_{F C}(\mathrm{~s})$ & $t_{T 1}(\mathrm{~s})$ & $t_{T 2}(\mathrm{~s})$ \\
\hline 14 & 98 & 3028 & 57 \\
\hline 5 & 43 & 1059 & 13 \\
\hline
\end{tabular}

Table 2. Comparison of the classification computing time with reference to the features set dimension.

In Table. 3., we present the results achieved with the selected 5 attributes determined by the SFFS technique. Besides, we compare the obtained classification accuracy on a test set of new dermatoscopic images, while using 14 features (subsection 3.2.1.), 5 features (subsection 3.2.2) and using the 9 features used in our previous work ([34]). Accuracy of the classification on the test set is evaluated in terms of "true-positive" (TP) rate (called also sensitivity) and "true-negative" (TN) rate (called also specificity).

\begin{tabular}{lll}
\hline features set dimension & $\mathrm{TP}(\%)$ & $\mathrm{TN}(\%)$ \\
\hline 14 & 71.3 & 93.5 \\
\hline 9 & 75.1 & 83.1 \\
\hline 5 & 74.8 & 80.6 \\
\hline
\end{tabular}

Table 3. Comparison of the classification accuracy with reference to the features set dimension.

At the end, we can conclude that the step of features selection with the SFFS technique allows us to reduce the number of features to five. This step allows to reduce considerably the dimension of the lesion's vector without significant loss of information about the lesion malignancy. In fact, the system with only five features permits to obtain a correct classification rate of $77.7 \%$ (with true-positive rate of $74.8 \%$ and true-negative rate of $80.6 \%$ ) in a very acceptable CPU time (less than one minute for both steps of feature calculation and of the lesion screening). The recorded correct classification rates are very comparable with the detection rates recorded with 9 features $(79.1 \%$ of correct classification rate with true-positive rate of $75.1 \%$ and true-negative rate of $83.1 \%$ ) and also with 14 features $(82.4 \%$ of correct classification rate with true-positive rate of $71.3 \%$ and truenegative rate of $93.5 \%$ ). However, the relative reduction of the number of features describing the lesion malignancy reduces the CPU time of a complete diagnosis of a lesion for about $50 \%$ (198 seconds with 14 features vs. 99 seconds with 5 features) and reduces the training time of the multi-layer perceptron for about $65 \%(3028$ seconds with 14 features v.s 1059 seconds with 5 features). 


\section{Conclusion and Future Work}

Different sections of this paper proposed solutions to specific problems related to computeraided diagnosis of pigmented skin lesions. Thus, an accelerated system for melanoma diagnosis was presented. In this study, we focus particularly on the important step of feature selection by modeling the clinical signs of lesions malignancy to enable the classification into either benign or malignant lesions class. Indeed, a large set of features was investigated by the application of feature selection techniques. The number of features relevant for classification was optimised and five well-selected features were sufficient to cover the major of discriminatory information about lesions malignancy. Results on real data proved the effectiveness of the proposed system by recording a good classification rate of $77.7 \%$ in a very encouraging computing time.

However, while the lesion detection problem is practically resolved, there is still much to do with regard to the extraction of diagnosis features. For example, we think to use a larger set of features as the input set for the subset feature selection techniques. This will probably permit to deduce more generalized descriptors for the lesion malignancy. Besides, we plan to use the genetic algorithms (GA) techniques for the subset feature selection with larger range of possible dimensions for the subset of features. Genetic algorithms can be used to explore the space of all possible subsets of features, so as to obtain, in the minimum of computing time, a set of features which optimise predicitive accuracy for the melanoma diagnosis.

\section{Acknowledgment}

The authors would like to thank sincerely the anonymous reviewers of this paper who made many important suggestions and helpful contributions that enabled us to improve the quality of the paper.

\section{References}

[1] American Cancer Society, www. cancer.org, (2002).

[2] A. BRESLOW, Thickness, cross-sectional area and depth of invasion in the prognosis of cutaneous melanoma, Annals of Surgey, 172(5), pp. 902-908, (1970).

[3] G. BURG, Das Melanom, Serie Gesundheit: Piper/VCH, (1993).

[4] T.E. CAMPOS, R.S. FERISAND AND R.M. CESAR, Improved face $\mathrm{x}$ non-face discrimination using Fourier descriptors through feature selection, $13^{\text {th }}$ Brazilian Symposium on Computer Graphics and Image Processing, Gramado, Brazil, (2000).

[5] T.M. COVER, The best two independent measurements are not the best, IEEE Transactions on Systems, Man and Cybernetics, SMC, 4(1), pp. 116-117, (1974).

[6] R. CuCChiara And C. GRANA, Using the topological tree for skin lesion structure description. Knowledge-Based Intelligent Information Engineering Systems and Allied Technologies (KES 2002 ), Frontiers in artificial intelligence and applications, IOS Press, 82(1), pp. 166-170, (2002).

[7] F. Ercal, A. ChaWla, W.V. StOecKer, H. LEE AND R.H. MOSS, Neural Network diagnosis of malignant melanoma from color images, IEEE Transactions on Biomedical Engineering, 41(9), pp. 837-845, (1994).

[8] S. FISHER, F. SCHMID AND J. GuILlOD, Analysis of skin lesions with pigmented networks, International conference on Image Processing (ICIP'96), Lausanne, Switzerland, (1996).

[9] R.J. Friedman, D.D Rigel AND A.W. Kopf, The continued importance of early detection of malignant melanoma, $C A, 41$, pp. 201-226, (1999).

[10] K.I. FunASHASHI, On the approximate realization of continous mappings by neural networks, International Journal of Neural Networks, pp. 183-192, (1989).

[11] H. Ganster, R. Rohrer, L. Paletta, T. Ebner, A. PINZ AND M. BINER, Comparison of neural networks and statistical methods in melanoma classification, Proc. $22^{\text {nd }}$ OAGM/AAPR Workshop, Illmitz, Austria, (1998).

[12] H. Ganster, A. Pinz, R. Rohrer, E. WildLing, M. BINDER AND H. KITTLER, Automated Melanoma Recognition, IEEE Transactions on Medical Imaging, 20(3), pp. 233-239, (2001).

[13] R.C. GonZALEZ AND R. E. Woods, Digital Image Processing, Adisson-Wesley, Reading, MA, second edition, (1992). 
[14] G.A. HANCE, S.E. UMBAUgh, W.V. STOECKER AND R.H. Moss, Unsupervised color image segmentation: With application to skin tumor borders, IEEE Engineering in Medicine and Biology Magazine, Vol 15, pp. 104-111, (1996).

[15] M. HinTZ-MADSEN, A probabilistic frame-work for classification of dermatoscopic images, ph.D. thesis, IMM, (1998).

[16] A.K. JAIN AND D. ZARGKER, Feature selection: Evaluation, Application and Small Sample Performance, IEEE Trans on Pattern Analysis and Machine Intelligence, 19 (2), pp. 153-158, (1997).

[17] L. KAUFMAN AND P.J. RoUSSEEUW, Finding groups in data: An introduction to cluster analysis, New York, USA, (1990).

[18] I. KhanfiR, A. Kallel, K. TaOuil, M. S. BouHLEL AND L. KAMOUN, Segmentation d'images par seuillage d'histogramme: Application Ó l'analyse des mÜlanomes, JTEA 2002, Tunisia, (2002).

[19] P.L. LANZI, Fast feature selection with genetic algorithms: a filter approach, IEEE International Conference on Evolutionary Computation (ICEC97), pp. 537-540, (1997).

[20] T. LeE, V. NG, D. MClean, A. Coldman, R. GALLAGHER AND J. SALE, A multi-stage segmentation method for images of skin lesions, IEEE Pacific Rim Conference on Communications, Computers and Signal Processing, pp. 602-605, (1995).

[21] T. LeE, V. Ng, R. Gallagher, A. Coldman and D. MCLEAN, Dullrazor: A software approach to hair removal from images, Computer in Biology and Medicine, 27, pp. 533-543, (1997).

[22] E. LeFEVRe, O. COLOT AND P. VANNOORENBERGHE, A classification method based on the DempsterShafer's theory and information criteria, FUSION'99, California, USA, (1999).

[23] M. LoÈVE, Fonctions alÚatoires de second ordre, In P. Levy, editor, Processus stochastiques et mouvement brownien, Hermann Eds, (1998).

[24] M.C. MinM, A.J. Sober AND T.B. FitzPATRICK, Primary melanoma of the skin: recognition and management, Journal of the American Academy of Dermatology, 2(3), pp. 179-197, (1980).

[25] P. Pudil, J. Novoviovca And J. KitTler, Floating search methods in feature selection, Pattern recognigion letters, 15, pp. 1119-1125, (1994).

[26] R. Rohre, H. Ganster, A. Pinz And M. Binder, Feature selection in melanoma recognition, ICPR'98, 2, pp. 1668-16670, (1998).

[27] T. Ross, H. Handels, J. Kreusch, H. Busche, H.H. WOLF AND S.J. POPPL, Automatic classification of skin tumors with high resolution surface profiles, Computer Analysis and Patterns, pp. 460 $465,(1995)$.
[28] A.J. Round, A.W. Duller AND P.J. Fish, Colour segmentation for lesion classification, $19^{\text {th }}$ International Conference - IEEE/EMBS, Chicago, USA, pp. 582-585, (1997).

[29] P. SCHMID-SAugeON, J. GuILlOD AND J.P. THIRAN Towards a computer-aided diagnosis system for pigmented skin lesions, Computerized Medical Imaging and Graphics, Vol 27, pp. 65-78, (2003).

[30] A. Sboner, E. Blanzieri, C. Eccher, P. BAuer, M. CRistofolini, G. Zumiani AND S. Forti, A knowledge based system for early melanoma support, Intelligent Data Analysis in Medicine and Pharmacology, UK, (2001).

[31] P. Somol, P. Pudil, J. Novovicova AND P. PACliK, Adaptive floating search methods in feature selection, Pattern recognigion letters, 20, pp. 1157-1163, (1999).

[32] H. VAFAIE AND K.D. JONG, Genetic algorithms as a tool for feature selection in machine learning, Proceedings of the $4^{\text {th }}$ International Conference on Tools with Artificial Intelligence, Alington, USA, (1992).

[33] P. VAnnoorenberghe, O. Colot And D. DE BRUCQ, Dempster-Shafer's theory as an aid to color information processing: Application to melanoma detection in dermatology, $10^{\text {th }}$ International Conference on Image Analysis and Processing, Italy, (1999).

[34] E. ZAGROUBA AND W. BARHOUMI, A prelimary approach for the automated recognition of malignant melanoma, Image Analysis and Stereology Journal, 23(2), pp. 121-135, (2004).

Received: August, 2004 Accepted: November, 2004

Contact address:

Ezzeddine Zagrouba Dèpartement des Sciences de l'Informatique Facultè des Sciences de Tunis 1060, Tunis, Tunisia e-mail: ezzeddine.zagrouba@f sm.rnu.tn

EZZEDDINE ZagrouBA received his $\mathrm{PhD}$ from the Polytechnic National Institute of Toulouse (France) in 1994. Since 1995, he is a Computer Sciences Professor in FSM (Facultè des Sciences de Monastir) and in FST (Facultè des Sciences de Tunis). His research within the laboratory LIP2-FST is focused on image analysis and computer vision.

WALID BARHOUMI received his Master's degree from the Polytechnic National Institute of Toulouse (France) in 1998. Currently, his is preparing a $\mathrm{PhD}$ thesis in computer sciences at the ENSI (Ecole Nationale des Sciences Informatiques de Tunis). His research within the laboratory CRISTAL-GRIFT is focused on image analysis and computer vision. 